\title{
Endothelial glycocalyx conditions influence nanoparticle uptake for passive targeting
}

\author{
This article was published in the following Dove Press journal: \\ International Journal of Nanomedicine \\ 21 July 2016 \\ Number of times this article has been viewed
}

\section{Ming J Cheng' \\ Rajiv Kumar ${ }^{2}$ \\ Srinivas Sridhar ${ }^{1-3}$ \\ Thomas J Webster ${ }^{1,4}$ \\ Eno E Ebong'}

'Department of Chemical Engineering, ${ }^{2}$ Department of Physics, Northeastern University, ${ }^{3}$ Department of Radiation Oncology, Harvard Medical School, Boston, MA, USA; ${ }^{4}$ Center of Excellence for Advanced Materials Research, King Abdulaziz University, Jeddah, Saudi Arabia

Video abstract

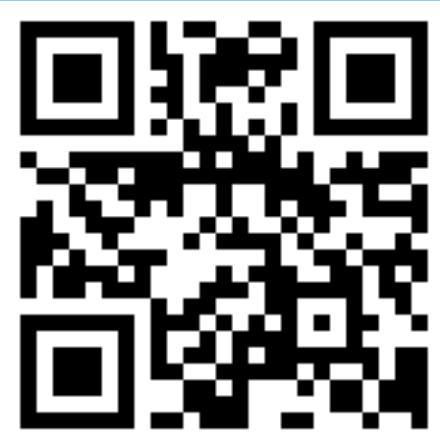

Point your SmartPhone at the code above. If you have a QR code reader the video abstract will appear. Or use: http://youtu.be/lAppq0F7 h4

Correspondence: Eno E Ebong Department of Chemical Engineering, Northeastern University, 360 Huntington Avenue, 3 I 3 Snell, Boston, MA 021 I5, USA

Tel +l 6173738744

Fax +I 6173732209

Email e.ebong@neu.edu
Abstract: Cardiovascular diseases are facilitated by endothelial cell (EC) dysfunction and coincide with EC glycocalyx coat shedding. These diseases may be prevented by delivering medications to affected vascular regions using circulating nanoparticle (NP) drug carriers. The objective of the present study was to observe how the delivery of $10 \mathrm{~nm}$ polyethylene glycol-coated gold NPs (PEG-AuNP) to ECs is impacted by glycocalyx structure on the EC surface. Rat fat pad endothelial cells were chosen for their robust glycocalyx, verified by fluorescent immunolabeling of adsorbed albumin and integrated heparan sulfate (HS) chains. Confocal fluorescent imaging revealed a $\sim 3 \mu \mathrm{m}$ thick glycocalyx layer, covering $75 \%$ of the ECs and containing abundant HS. This healthy glycocalyx hindered the uptake of PEG-AuNP as expected because glycocalyx pores are typically $7 \mathrm{~nm}$ wide. Additional glycocalyx models tested included: a collapsed glycocalyx obtained by culturing cells in reduced protein media, a degraded glycocalyx obtained by applying heparinase III enzyme to specifically cleave HS, and a recovered glycocalyx obtained by supplementing exogenous HS into the media after enzyme degradation. The collapsed glycocalyx was $\sim 2 \mu \mathrm{m}$ thick with unchanged EC coverage and sustained HS content. The degraded glycocalyx showed similar changes in EC thickness and coverage but its HS thickness was reduced to $0.7 \mu \mathrm{m}$ and spanned only $10 \%$ of the original EC surface. Both dysfunctional models retained six- to sevenfold more PEG-AuNP compared to the healthy glycocalyx. The collapsed glycocalyx permitted NPs to cross the glycocalyx into intracellular spaces, whereas the degraded glycocalyx trapped the PEG-AuNP within the glycocalyx. The repaired glycocalyx model partially restored HS thickness to $1.2 \mu \mathrm{m}$ and $44 \%$ coverage of the ECs, but it was able to reverse the NP uptake back to baseline levels. In summary, this study showed that the glycocalyx structure is critical for NP uptake by ECs and may serve as a passive pathway for delivering NPs to dysfunctional ECs.

Keywords: glycocalyx, heparan sulfate, endothelial cells, NP, gold

\section{Introduction}

The endothelium lines the blood vessel interior and regulates blood vessel stiffness for healthy cardiovascular function. ${ }^{1}$ It also acts as a barrier between the blood and underlying tissue by filtering molecules and cells that seek to cross between the two compartments. ${ }^{1}$ These are just a few of the known roles of the endothelium. Its proper function relies on its glycocalyx coating that consists of a glycosaminoglycan and proteoglycan mesh layer. This porous structure greatly restricts the passage to plasma proteins such as albumin of size $7 \mathrm{~nm}$, as well as smaller solutes and water molecules. ${ }^{2,3}$ The glycocalyx glycosaminoglycans, the most common of which is heparan sulfate (HS), ${ }^{4-6}$ are anchored to endothelial cells (ECs) via transmembrane proteoglycans. $^{7}$ Sialic acid is another glycosaminoglycan of the glycocalyx that is integral in the cellular-molecular interactions, while hyaluronic acid and its receptors, 
such as CD44, are well characterized and important in cellular function and communication. ${ }^{7}$ The glycosaminoglycans also interact with plasma proteins and other blood-borne molecules, producing variable glycocalyx thicknesses on the outer EC surface. ${ }^{2,8}$ Additionally, the glycocalyx mediates EC signaling and remodeling to provide upstream control of the aforementioned vascular stiffness regulation and filtration roles through its attachment to the EC membrane. As a result, the ECs and the glycocalyx cooperate to exhibit protective and regulatory functions in vascular health. ${ }^{1,9-12}$ Vascular disease progression, such as atherosclerotic vessel hardening or lipid- and macrophage-filled plaque formation, is facilitated by EC dysfunction ${ }^{13}$ and coincides with shedding of the EC glycocalyx coat. ${ }^{14-17}$ Furthermore, a compromised glycocalyx has been shown to increase the permeability of nanoscale particles in other cell types such as erythrocytes ${ }^{18}$ and cancer tumor cells. ${ }^{19}$ Therefore, a potential avenue for treating vascular diseases is to target ECs and their glycocalyx, rather than simply utilizing the systemic and medicinal treatments that are common today. ${ }^{20}$

EC-specific drug targeting can be enabled with the use of nanoparticle (NP)-based medicine, which has recently been a major focus for researchers. ${ }^{21,22}$ Studies have shown that nanosized particles can be fine-tuned to deliver drugs and control a multitude of scenarios, including extending circulation time in the blood stream and targeting specific organs or sites of disease. ${ }^{22,23}$ Lengthening NP residence times in the blood stream, through delaying clearance by the immune system or filtration by the kidneys, is achieved by covering the NPs with neutrally charged polymer coatings. ${ }^{23-27}$ While local injection of NP-based materials is available for joint and wound therapy, ${ }^{25,28,29}$ there are several common scenarios that require delivery to difficult-to-reach or unknown locations, such as tumors and metastatic disease. These situations often involve systemic administration through either oral ingestion or, relevant to the present study, venous injection. ${ }^{30}$ Such approaches require that the surface of NPs be conjugated to targeting ligands to increase the odds of particle accumulation at the desired location. ${ }^{26}$ In addition, this targeting strategy typically leverages the overexpression of enzymes, decrease in $\mathrm{pH},{ }^{26,31,32}$ leakiness of the tissue, and/or enhanced tissue retention of the nanosized particles. ${ }^{23,29}$ These features can be exploited to activate the circulating NP drug carriers at the target organ or disease site. ${ }^{33,34}$ The presence and integrity of the glycocalyx coating of the EC play a critical role in the cellular uptake of circulating NPs. Unfortunately, the glycocalyx is generally not properly modeled in the development of NPbased therapeutic approaches and can lead to incorrect uptake and permeability measurements in vitro. ${ }^{18,19,28}$ Additionally, ECs are not the only cell type to express glycocalyx - most other mammalian cells as well as circulating cancer cells also express unique glycocalyx..$^{18,19,28,33,35}$ Therefore, elucidating the role the glycocalyx plays in the cellular uptake of NPs by ECs will benefit the field of nanomedicine and drug delivery for cardiovascular and other diseases.

This study investigated whether EC exclusion and uptake of polymer-coated NPs are determined by the state of the glycocalyx. The glycocalyx thickness and the extent to which it covers monolayers of EC were measured by fluorescent immunolabeling of glycocalyx components: adsorbed bovine serum albumin (BSA) and HS. This was followed by confocal imaging and analysis, where an untreated intact glycocalyx, protein deficiency-induced collapsed glycocalyx, enzymatically degraded glycocalyx, and repaired glycocalyx were examined. For these glycocalyx configurations, EC uptake of fluorescent polyethelyne glycol (PEG)-ylated gold NPs (PEG-AuNP) ${ }^{36}$ was assessed by quantifying and mapping the subcellular locations of the fluorescent signals emitted by the NPs. The exclusion of PEG-AuNP by healthy and recovered glycocalyx and an increased uptake under glycocalyx dysfunction confirm that glycocalyx health is a critical factor for designing NP drug delivery therapeutics.

\section{Materials and methods RFPEC culture}

A rat fat pad endothelial cell (RFPEC) line $^{37}$ was utilized, originally isolated from rat epididymal fat pads by Dr Mia Thi of Albert Einstein College of Medicine (Bronx, NY, USA). RFPECs were selected for their ability to naturally express a robust glycocalyx. ${ }^{38}$ These ECs were cultured in tissue culture flasks at $37^{\circ} \mathrm{C}$ and $5 \% \mathrm{CO}_{2}$ in a humidified environment with Dulbecco's Modified Eagle's Medium (DMEM) supplemented with 10\% fetal bovine serum (FBS) and 1\% penicillin-streptomycin, all purchased from Thermo Fisher Scientific (Waltham, MA, USA). For 3-(4,5-dimethylthiazol2-yl)-5-(3-carboxymethoxyphenyl)-2-(4-sulfophenyl)-2Htetrazolium (MTS) assays (Sigma-Aldrich Co., St Louis, MO, USA), RFPECs were seeded at $3.7 \times 10^{4}$ cells $/ \mathrm{cm}^{2}$ in tissue culture-treated, 96-well plates (Falcon; Thermo Fisher Scientific) and imaged on a SpectraMax M3 plate reader to show cell viability and metabolic activity. For confocal imaging, RFPECs were seeded onto $12 \mathrm{~mm}$ diameter round glass cover slips (Thermo Fisher Scientific) at $1 \times 10^{4}$ cells $/ \mathrm{cm}^{2}$ in tissue culture-treated six-well plates (Falcon; Thermo Fisher Scientific). In both cases, the cells were allowed to 
grow for $48-60$ hours to full confluence, before starting the experiment.

\section{PEG-AuNP}

Gold was chosen to be the core of the NPs for its inert property and its widespread use in other applications, such as imaging under magnetic resonance imaging, surfaceenhanced Raman scattering, and radiotherapy. ${ }^{39-41}$ AuNP functionalized with a polyethylene glycol (PEG) corona were synthesized following the protocols reported by Kumar et al. ${ }^{36}$ Following the formation of tetrakis-(hydroxymethyl)-phosphonium chloride-coated AuNP (Sigma-Aldrich Co.), three types of functional PEG were added onto its surface: $\mathrm{SH}-$ PEG-OCH ${ }_{3}, \mathrm{SH}-\mathrm{PEG}-\mathrm{COOH}$, and $\mathrm{SH}-\mathrm{PEG}-\mathrm{NH}_{2}$ (Laysan Bio, Arab, AL, USA) at a ratio of 1:1:2 using gold-thiol chemistry and confirmed using Fourier transform infrared spectroscopy (Bruker Vertex-70; Bruker Optik GmbH, Ettlingen, Germany). The variety of functional PEG allows for additional customization in future iterations of the NPs. After dialysis in 12-14 $\mathrm{kDa}$ molecular weight cutoff tubes (EMD Millipore, Billerica, MA, USA) and lyophilization, an Alexa Fluor 647 N-Hydroxysuccinimide (NHS) ester (succinimidyl ester) was conjugated onto the $\mathrm{NH}_{2}$ functional groups on the AuNP to allow for visualization under fluorescence. NP size was determined using transmission electron microscope (TEM) (JEOL JEM-1000, Tokyo, Japan) at $80 \mathrm{kV}$ and $100,000 \times$ magnification and dynamic light scattering (DLS) (Brookhaven 90Plus Particle Size
Analyzer; Brookhaven Instruments Corporation, Holtsville, NY, USA). PEG-AuNP fluorescence activity was confirmed using Jobin Yvon Fluoromax 4.

\section{RFPEC glycocalyx treatments}

Table 1 summarizes how the RFPECs are cultured to generate various glycocalyx configurations. For control glycocalyx conditions, RFPEC cultures were grown in DMEM with 10\% FBS and $1 \%$ penicillin-streptomycin as described earlier. To generate dysfunctional glycocalyx conditions, the regular culture media of other RFPEC cultures was replaced with two variations of the modified media. For the collapsed glycocalyx configuration, the cells were grown in low serum media that consisted of DMEM with $1 \%$ penicillin-streptomycin and only $2 \%$ FBS. $^{1}$ For the degraded glycocalyx configuration, cultures were pretreated with DMEM/10\% FBS/1\% penicillin-streptomycin containing a $2.5 \times 10^{-6} \mathrm{IU}$ heparinase III (Hep III) (IBEX Pharmaceutical, Montreal, QC, Canada) enzyme for 2 hours, selectively degrading the HS glycosaminoglycans of the glycocalyx. To simulate a repaired glycocalyx state, the media containing enzyme was replaced with exogenous HS (Celsus Laboratories, Cincinnati, $\mathrm{OH}$ USA) dissolved in DMEM/10\% FBS/1\% penicillin-streptomycin at $59 \mu \mathrm{g} / \mathrm{mL}$. All RFPEC cultures, including those with untreated, collapsed, degraded, or repaired glycocalyx configurations, were exposed to $550 \mu \mathrm{g} / \mathrm{mL}$ of lyophilized and ultraviolet-sterilized PEG-AuNP during the 16-hour treatment time. Afterward, the free floating particles were

Table I Treatment details and media composition for glycocalyx configurations

\begin{tabular}{|c|c|c|c|c|}
\hline & \multicolumn{4}{|c|}{ Glycocalyx configurations } \\
\hline & $\begin{array}{l}\text { Control } \\
\text { (untreated) }\end{array}$ & $\begin{array}{l}\text { Collapse } \\
\text { (low serum) }\end{array}$ & $\begin{array}{l}\text { Degraded } \\
\text { (Hep III) }\end{array}$ & $\begin{array}{l}\text { Repaired } \\
\text { (Hep III + HS) }\end{array}$ \\
\hline \multicolumn{5}{|l|}{ Culture } \\
\hline DMEM + I\% P/S & $x$ & $x$ & $x$ & $x$ \\
\hline $10 \% \mathrm{FBS}$ & $\mathrm{X}$ & $x$ & $\mathrm{x}$ & $\mathrm{x}$ \\
\hline \multicolumn{5}{|l|}{ Pretreatment } \\
\hline DMEM + I\% P/S & $x$ & $x$ & $x$ & $x$ \\
\hline $10 \% \mathrm{FBS}$ & $x$ & & $x$ & $x$ \\
\hline $2 \% \mathrm{FBS}$ & & $x$ & & \\
\hline $2.5 \times 10^{-6} \mathrm{IU} \mathrm{Hep}$ III & & & $x$ & $x$ \\
\hline \multicolumn{5}{|l|}{ Treatment } \\
\hline DMEM + I\% P/S & $x$ & $x$ & $\mathrm{x}$ & $x$ \\
\hline $10 \% \mathrm{FBS}$ & $x$ & & $x$ & $x$ \\
\hline $2 \% \mathrm{FBS}$ & & $x$ & & \\
\hline $59 \mu \mathrm{g} / \mathrm{mL} \mathrm{HS}$ & & & & $\mathrm{X}$ \\
\hline $550 \mu \mathrm{g} / \mathrm{mL}$ PEG-AuNP & $\mathrm{x}$ & $x$ & $x$ & $x$ \\
\hline
\end{tabular}

Note: The $\mathrm{X}$ is to indicate the component on the left hand column is present within each of the configuration media.

Abbreviations: HEP III, heparinase III; HS, heparan sulfate; DMEM, Dubecco's Modified Eagle's Medium; P/S, penicillin/streptomycin; FBS, fetal bovine serum; PEG-AuNP, PEGylated gold NPs. 
gently washed out before fixation to ensure that only the AuNP entrapped within the glycocalyx would be imaged. The red fluorescence detected from conjugated Alexa Fluor 647 was attributed to AuNP within the RFPEC monolayers (details are given in the "Confocal imaging and analysis" section).

\section{Immunostaining}

To visualize the glycocalyx, BSA from the media or the HS glycosaminoglycan was fluorescently tagged through immunostaining. RFPECs were first rinsed in phosphate buffered saline (PBS) (Corning Incorporated, Corning, NY, USA) containing $\mathrm{Ca}^{2+}$ and $\mathrm{Mg}^{2+}$ adjusted to a $\mathrm{pH}$ of 7.2. RFPEC slides were then fixed with $2 \%$ paraformaldehyde $/ 0.1 \%$ gluteraldehyde (Electron Microscopy Sciences, Hatfield, PA, USA) in PBS for 30 minutes. PBS was used to thoroughly wash the fixatives before the slides were blocked with $2 \%$ goat serum (Sigma-Aldrich Co.) diluted in PBS for 30 minutes. Then, $0.02 \mu \mathrm{g} / \mu \mathrm{L}$ of the primary antibody in PBS containing 2\% goat serum was introduced and left in a humidity-controlled chamber at $4^{\circ} \mathrm{C}$, overnight for BSA staining or for three nights for HS staining. The primary BSA stain utilized a rabbit IgG anti-albumin (bovine serum), obtained from Thermo Fisher Scientific, and the primary HS stain used a 10E4 epitope HS mouse monoclonal IgM antibody from Amsbio (Abingdon, UK). Following incubation with primary antibody, RFPECs were washed again in PBS and then incubated in $0.002 \mu \mathrm{g} / \mu \mathrm{L}$ of secondary antibody in PBS containing 2\% goat serum for 30 minutes at room temperature and in the dark. Alexa Fluor 488 goat anti-rabbit IgG and Alexa Fluor 488 goat anti-mouse IgG, IgM fractions (Thermo Fisher Scientific) were used for the secondary detection of BSA and HS stains, respectively. After incubation with secondary antibody, the RFPECs were washed in PBS and then mounted onto glass microscope slides (Thermo Fisher Scientific) using VECTASHIELD mounting medium (Vector Laboratories, Burlingame, CA, USA). The mounting medium contained 4',6-diamidino-2-phenylindole (DAPI), a fluorescent stain that binds to adenine and thymine regions of DNA to mark the cell nuclei. Generic nail polish was used to seal the slides before imaging.

\section{Confocal imaging and analysis}

A Zeiss LSM 700 confocal microscope (Carl Zeiss Meditec AG, Jena, Germany) using a $63 \times$ oil immersion objective with excitation wavelengths of 350,490 , and $650 \mathrm{~nm}$ were used to obtain XY-plane slices of the nuclei of RFPECs, fluorescent glycocalyx (BSA and HS), and PEG-AuNP within RFPECs, respectively. Slices were captured at intervals of $0.2 \mu \mathrm{m}$ and assembled into Z-projections using NIH ImageJ software (ImageJ version 1.49k; National Institute of Health, USA). Glycocalyx coverage of RFPEC layers was quantified using ImageJ to analyze the en face view of the Z-projections of green channel $(490 \mathrm{~nm})$. The fluorescence threshold was adjusted to eliminate the background noise and then converted to black and white such that the green signal became white and the background signal was reduced to black. ImageJ measured the percentage of white areas compared to the total field of view in the micrograph, which was then equated to RFPEC glycocalyx coverage. Glycocalyx thickness was also measured with the help of ImageJ based on cross sections (orthogonal views) of the Z-projections of green channel. Three evenly distributed locations on the surface of each RFPEC were randomly selected by ImageJ. Lines perpendicular to the RFPEC surface were drawn at each point from the base of the glycocalyx to the top of the glycocalyx, as indicated by the green fluorescence. The length of the lines was measured and averaged to determine glycocalyx thickness.

It is important to note that BSA immunofluorescence was taken as an indicator of the structure of the whole glycocalyx, as albumin from the FBS in culture media is adsorbed throughout the glycocalyx. Therefore, it is reasonable to assume that BSA thickness and coverage measurements are equal to the overall glycocalyx thickness and coverage. On the other hand, HS immunofluorescence revealed thickness and coverage for the HS component only within the glycocalyx mesh, allowing for a more close examination during HS degradation and recovery. Red fluorescence, as detected by ImageJ, was attributed to clusters of the PEG-AuNP trapped within the glycocalyx. ${ }^{36}$ To quantify the amount of PEG-AuNP present in each sample, Z-stacks were projected en face and the number of red pixels was measured in ImageJ using the measure area tool. Additionally, orthogonal views were used to map the distributions of regions where the PEG-AuNP localized within the cells by using the plot profile function in ImageJ and correlating the height of red fluorescence in relation to glycocalyx position. DAPI staining of RFPEC nuclei was not quantified and only served to mark the RFPEC.

\section{Statistics}

NP uptake values, glycocalyx thickness, and percent coverage measurements are expressed as mean \pm standard error of the mean. These measurements were taken from five independent experiments. For each condition studied, two samples were studied per experiment. From each sample, a minimum of five confocal Z-stacks were collected. Data set groups were individually compared against the data set of the 
untreated control group. The difference between the groups was analyzed for statistical significance using an unpaired Student's $t$-test with an alpha value of 0.05 .

\section{Results and discussion Characterization of fluorescent, PEG-AuNP}

Synthesized AuNP were imaged under TEM and DLS, giving a diameter of $2.8 \pm 0.8$ and $3.0 \pm 0.6 \mathrm{~nm}$, respectively (Figure 1A-C). After PEGylation, the diameter of the particles increased to $10.6 \pm 1.2 \mathrm{~nm}$ (Figure 1C). Furthermore, the particles were confirmed to have a fluorescence emission peak $\sim 667 \mathrm{~nm}$ (Figure 1D) and insignificant toxicity to RFPEC up to $1 \mathrm{mg} / \mathrm{mL}$ (Figure 1E). From zeta potential calculations, particle charge was found to be $-14 \mathrm{mV}$ (data not shown). The size of the PEG-AuNP is ideal for observing NP-EC interactions because of the $7 \mathrm{~nm}$ porosity of the glycocaly $x^{1}$ as well as the negatively charged glycocalyx surface. A healthy glycocalyx should prevent these particles from becoming entrapped, but once the glycocalyx is compromised and the barrier function is impaired, the PEG-AuNP will be able to pass through and be taken up into the cells. The Alexa Fluor 647 probes were imaged alongside the healthy, collapsed, degraded, and regenerated glycocalyx samples to observe total AuNP uptake as well as the distribution within the endothelial monolayer.

\section{Healthy glycocalyx excludes PEG-AuNP from ECs}

ECs with an intact and healthy glycocalyx were modeled by RFPECs and their unmanipulated glycocalyx. As reported by others, ${ }^{37,42}$ RFPECs grown in static culture express a robust glycocalyx, unlike other ECs whose glycocalyx development is hindered in static conditions. The dimensions of healthy RFPEC glycocalyx were obtained by measuring BSA coverage and thickness on the EC surface. As previously mentioned, the albumin from the culture media is adsorbed throughout the expanse of the glycocalyx, which gives it its full thickness. ${ }^{43}$ From confocal measurements of BSA immunofluorescence, the total glycocalyx in healthy conditions was found to have a thickness of $3.2 \pm 0.2 \mu \mathrm{m}$ and cover $75.2 \% \pm 4.0 \%$ (Figure 2 ) of the EC layer surface. Upon immunostaining and measurement of the HS subcomponent of the glycocalyx, it was found to be $1.8 \pm 0.2 \mu \mathrm{m}$ thick and to cover $75.2 \% \pm 10.6 \%$ of the RFPEC surface (Figure 3). As the most abundant

\section{A}
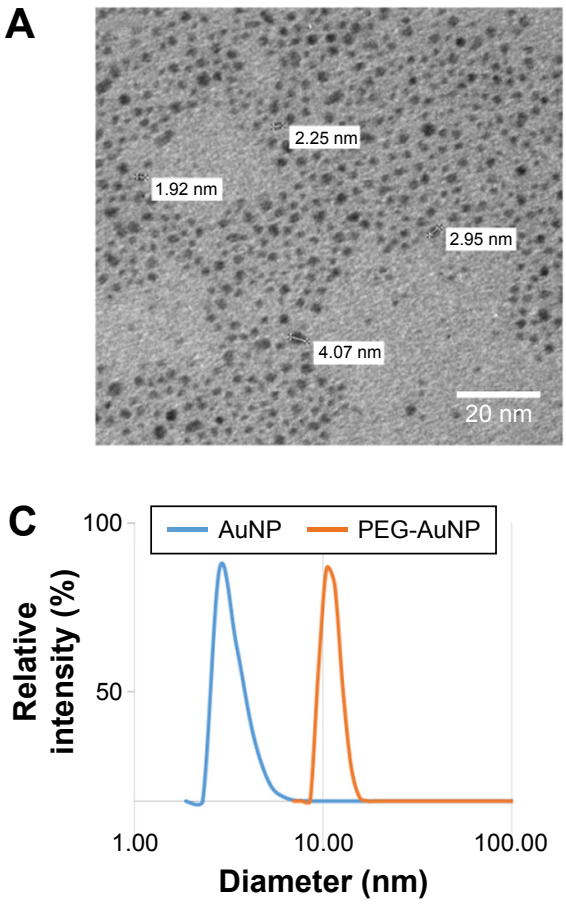

B
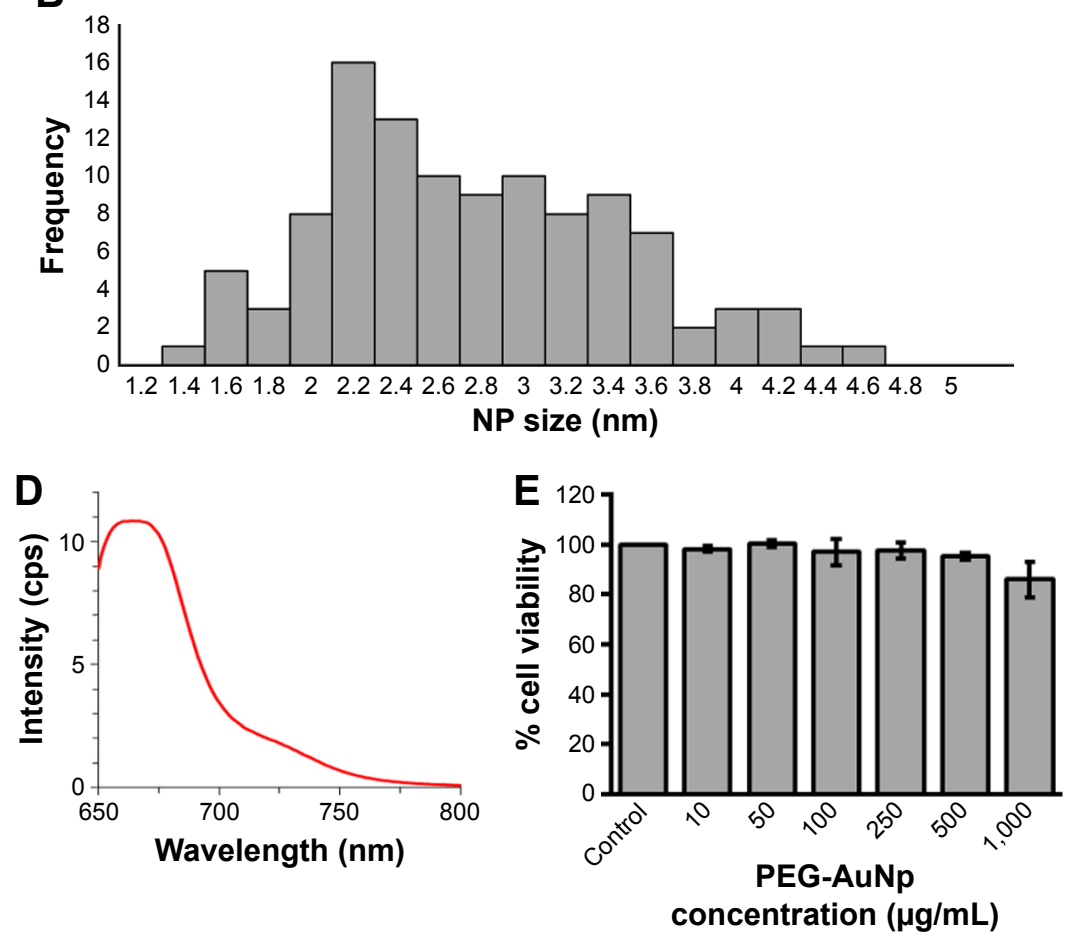

Figure I Size, fluorescence, and toxicity measurements of PEG-AuNP.

Notes: (A) TEM of AuNP showing individual NPs and sample measurements. (B) Histogram of AuNP size distribution measured from TEM images. (C) DLS plot indicating an increase in diameter of AuNP after PEGylation. (D) Fluorescence peak at $667 \mathrm{~nm}$ matches the emission of Alexa Fluor 647 conjugated to PEG-AuNP. (E) MTS assays show no PEG-AuNP toxicity to RFPEC up to $1,000 \mu \mathrm{g} / \mathrm{mL}$ for 16 hours.

Abbreviations: TEM, transmission electron microscope; AuNP, gold NPs; DLS, dynamic light scattering; PEG, polyethelyne glycol; MTS, 3-(4,5-dimethylthiazol-2-yl)-5(3-carboxymethoxyphenyl)-2-(4-sulfophenyl)-2H-tetrazolium; RFPEC, rat fat pad endothelial cell; NP, nanoparticle. 

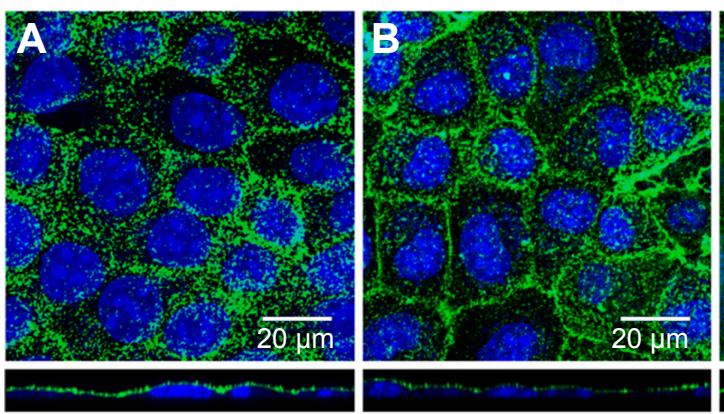

E

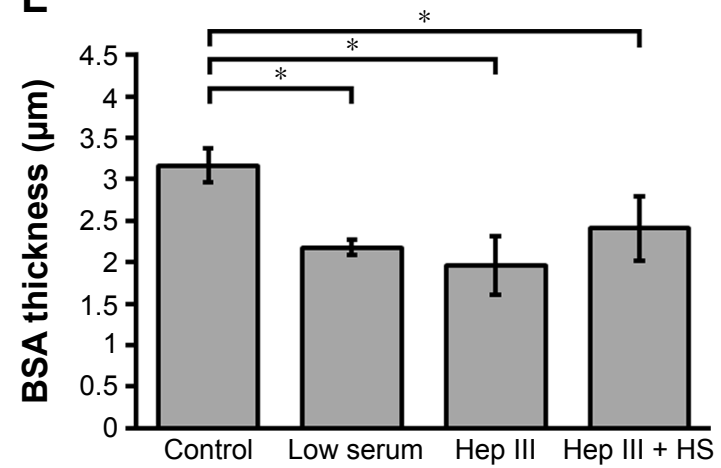

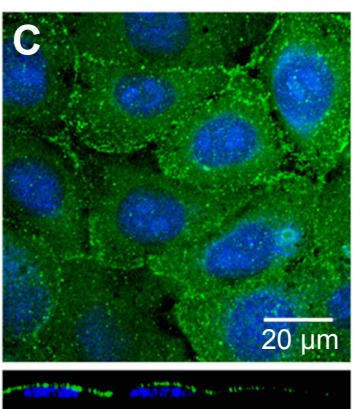

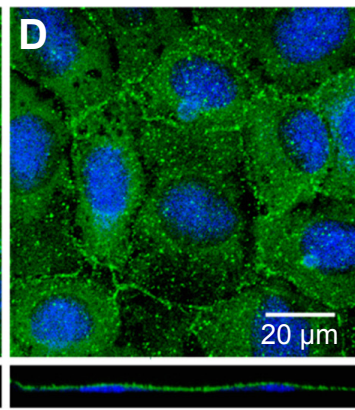

$\mathbf{F}$

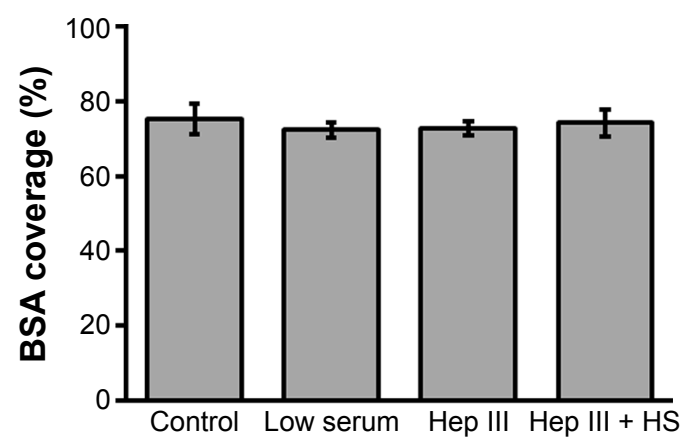

Figure 2 En face and cross sections of RFPEC in which BSA was immunostained in green and cell nuclei in blue.

Notes: (A) Control, (B) low serum, (C) Hep III, and (D) Hep III + HS. (E) Quantification of the overall thickness of glycocalyx after various treatments - control, low serum, Hep III, Hep III + HS - depicted by staining BSA adsorbed onto the glycosaminoglycan matrix. The glycocalyx treatments and recovery decreased overall thickness. (F) The glycocalyx coverage as seen by BSA immunofluorescence. The various treatments and glycocalyx conditions did not affect overall glycocalyx coverage. $* P<0.05$ versus control.

Abbreviations: RFPEC, rat fat pad endothelial cell; BSA, bovine serum albumin; HEP III, heparinase III; HS, heparan sulfate.
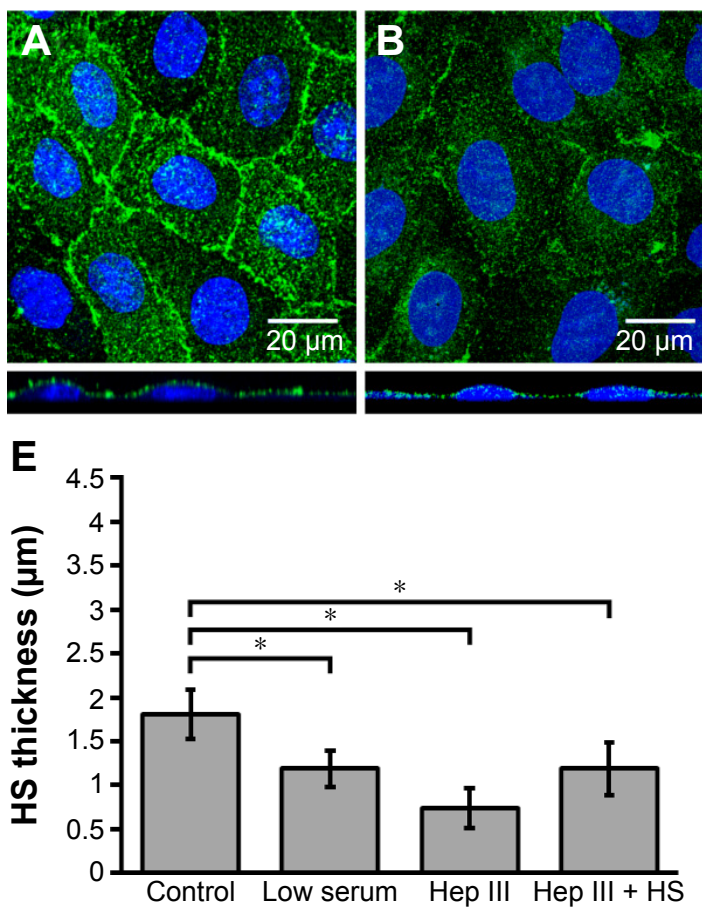
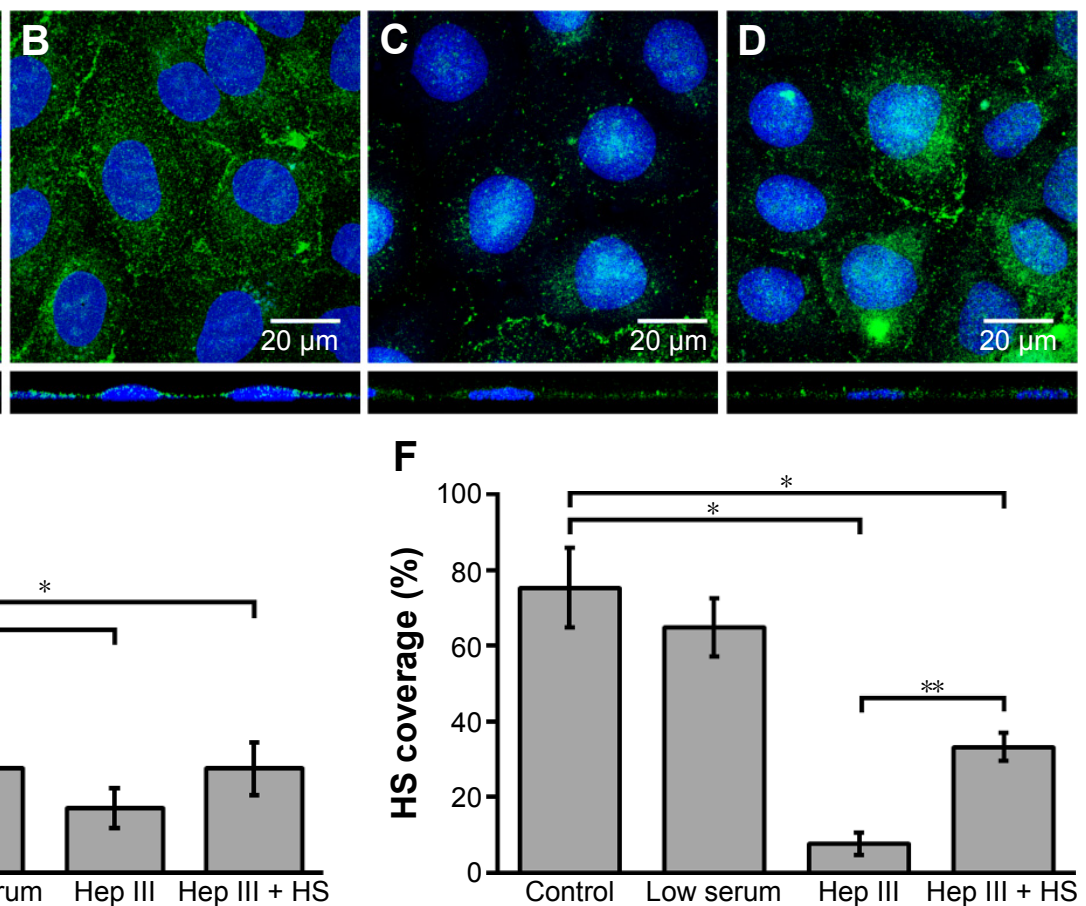

Figure 3 En face and cross sections of RFPEC in which HS was immunostained in green and cell nuclei in blue.

Notes: (A) Control, (B) low serum, (C) Hep III, and (D) Hep III + HS. (E) Thickness measurements of the glycocalyx when observing only the HS component by binding the IOE4 epitope HS antibody to the glycosaminoglycan under control, low serum, Hep III, and Hep III + HS treatments. HS thickness decreased significantly for all treatments. (F) HS coverage of RFPEC after various treatments. HS coverage decreased significantly after heparinase III treatment and recovered after $\mathrm{HS}$ addition. $* P<0.05$ versus control and $* * P<0.05$ versus Hep III.

Abbreviations: Hep III, heparinase III; HS, heparan sulfate; RFPEC, rat fat pad endothelial cell. 
glycosaminoglycan, HS appears to be integrated within the glycocalyx mesh close to the cell surface, as seen by the adjacent nuclei from the DAPI stain within the cross sections (Figure 3). Previous studies suggest that the outer region of the glycocalyx consists largely of hyaluronan and sialic acid, which are two other important components of the glycocalyx. ${ }^{10,18,34,44}$ These glycocalyx elements were not explored in the present study, although their roles and the roles of other glycosaminoglycans in EC permeability is a question of great interest. The dimensions of total glycocalyx (BSA) and the HS subcomponent measured were within the range of previously reported values for rodent cell cultures and animal models using the same fixation and microscopy approach..$^{37,38,45}$ Therefore, these results provide strong indication that the untreated RFPEC glycocalyx is healthy; owing to its substantial thickness and continuity, the RFPEC glycocalyx mesh is expected to serve as a robust, molecular sieve as seen in the body.

To confirm the barrier function of a healthy RFPEC glycocalyx, the extent of uptake of $10 \mathrm{~nm}$ diameter PEGAuNP ${ }^{36}$ was measured by the red fluorescence emitted by the conjugated Alexa Fluor 647. It was found to be strikingly low with two or three clusters of PEG-AuNP per cross section (Figure $4 \mathrm{~A}$ ), covering $1 \%$ of the image (assessed by counting pixels) and presenting a normalized value of $\sim 1$ in Figure 4B. The appearance of red particles in clusters can be attributed to the particles' tendency to aggregate after entrapment. The low uptake of these NP clusters demonstrates that, in healthy conditions, the glycocalyx does not allow many 10 $\mathrm{nm}$ NPs to penetrate into the endothelium. This NP exclusion is likely due to the $7 \mathrm{~nm}$ pore size of the glycocalyx. In addition, the NPs are negatively charged $(-14 \mathrm{mV})$, which cause the particles to be repelled by the negative charge of the glycocalyx. Identification of the red fluorescence location within the monolayer showed an even dispersion, indicating no specific localization within the cells (Figure 4C), for the small number of particles present.

\section{Glycocalyx dysfunctions lead to distinct PEG-AuNP uptake}

Next, the glycocalyx dysfunction treatments were applied to the EC to observe whether NP permeability was influenced
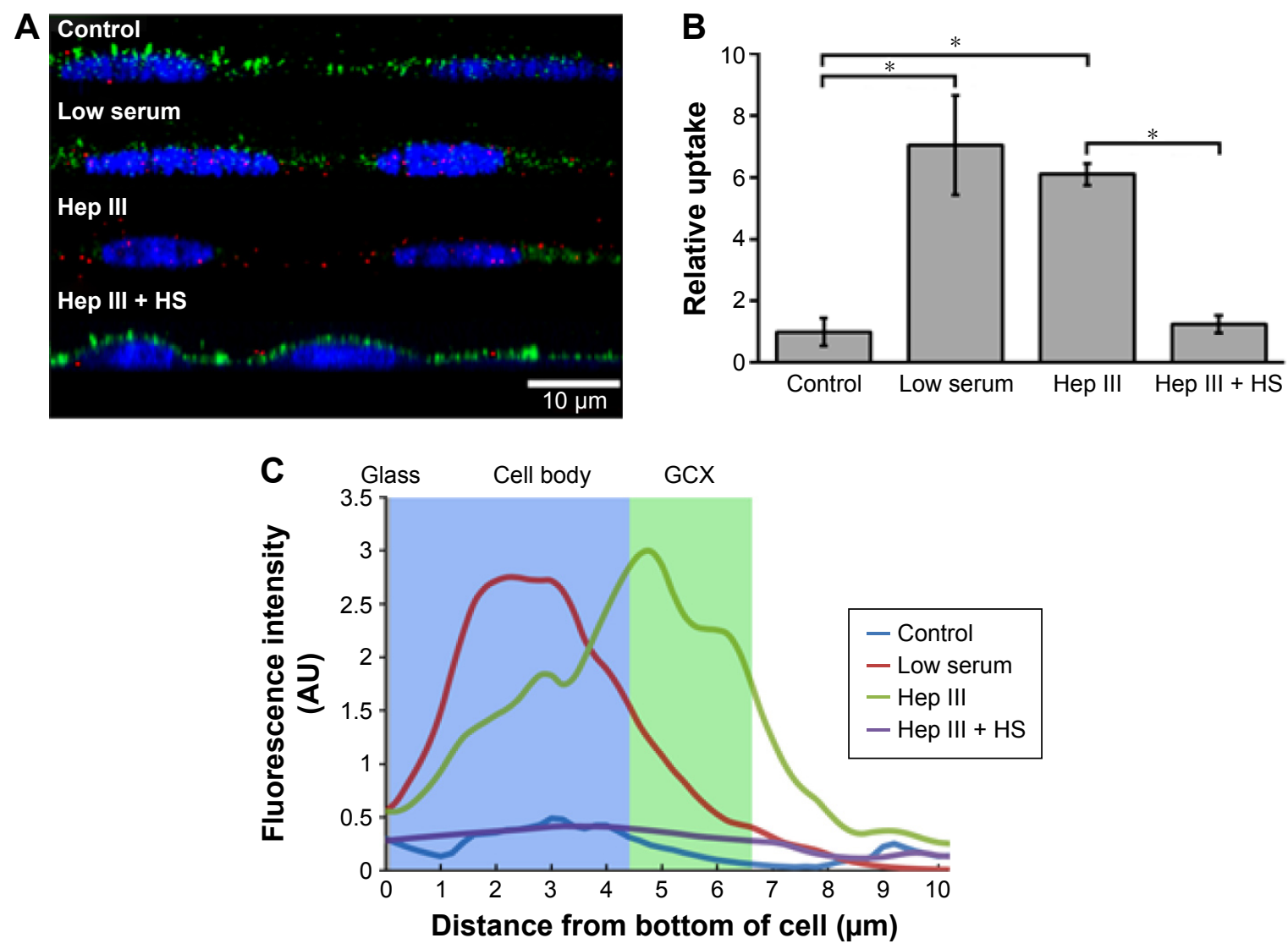

Figure 4 Uptake and localization differences between glycocalyx conditions.

Notes: (A) Confocal cross sections of PEG-AuNP (red) in RFPEC under various glycocalyx conditions. (B) Relative fluorescent AuNP uptake by quantifying the amount of red fluorescence in cross-sectional images and normalizing to controls. $* P<0.05$. (C) Localization of PEG-AuNP within the cross sections vary between dysfunctional types. Red fluorescence was measured with respect to cell height and consolidated in a histogram.

Abbreviations: AuNP, gold NPs; PEG, polyethelyne glycol; RFPEC, rat fat pad endothelial cell; Hep III, heparinase III; HS, heparan sulfate; GCX, glycocalyx. 
by changes in the glycocalyx. The first model of dysfunctional glycocalyx was in the collapsed form, generated by culturing RFPEC in a serum-deficient setting containing $2 \%$ FBS rather than the normal 10\%. For a collapsed glycocalyx, due to low serum treatment, total glycocalyx (BSA) thickness decreased by $31 \%$ from $3.2 \pm 0.2$ to $2.2 \pm 0.1 \mu \mathrm{m}$ (Figure $2 \mathrm{~B}$ and E). On the other hand, the coverage of the whole glycocalyx was not significantly affected at $72.3 \% \pm 2.0 \%$ compared to the control (75\%) (Figure 2F). Serum-starved, collapsed glycocalyx measurements for HS were comparable to those of the whole glycocalyx where HS thickness decreased by $34 \%$ to $1.19 \pm 0.2 \mu \mathrm{m}$ (Figure $3 \mathrm{~B}$ and $\mathrm{E}$ ), but its coverage of EC was not affected significantly at $64.8 \% \pm 7.6 \%$ (Figure 3F).

The second dysfunctional model of degraded glycocalyx was achieved through exposure of RFPEC to heparinase III for selective degradation of the HS component. Heparinase III treatment resulted in a whole glycocalyx (BSA) thickness of $1.96 \pm 0.3 \mu \mathrm{m}$ (Figure $2 \mathrm{C}$ and $\mathrm{E}$ ), a 38\% decrease from the control measurement of $3.2 \pm 0.2 \mu \mathrm{m}$. Heparinase III slightly disrupted the continuity of the whole glycocalyx, resulting in the coverage of $72.7 \% \pm 1.9 \%$ (Figure $2 \mathrm{C}$ and F) of the EC layer, a decrease of 3.4\%. We previously reported on the persisting structural stability of the whole glycocalyx, even after the enzymatic removal of individual glycosaminoglycans. ${ }^{38}$ However, the results from the current heparinase III experiment produced a decrease in total glycocalyx thickness but statistically negligible change in the continuity of the total glycocalyx. The discrepancy between our previous and presently reported measurements may have been due to the treatment time scale. The RFPECs in this study were exposed to 2 hours of enzyme treatment followed by another 16 hours of incubation with NPs to assess the uptake. Previously, we only exposed RFPEC to an enzyme for a short 2-hour period before immediately preserving the cells and immunostaining. ${ }^{38}$ Our present approach yielded a significantly thinner matrix while preserving the continuity of the total glycocalyx matrix. Focusing on the major glycocalyx subcomponent, HS, its thickness was statistically significantly decreased by $59 \%$ from $1.8 \pm 0.2$ to $0.74 \pm 0.2 \mu \mathrm{m}$ (Figure $3 \mathrm{C}$ and $\mathrm{E}$ ). This was expected due to the specific action of heparinase III and resulted in a substantial $89.8 \%$ decrease in HS coverage from $75.2 \% \pm 10.6 \%$ to $7.7 \% \pm 2.9 \%$ (Figure 3C and F).

The thinning of the glycocalyx through dysfunction may result in an increased permeability of the endothelial glycocalyx. Wiesinger et al reported an association between sickness and thinning of the glycocalyx, both in vivo (in septic mice) and in vitro (in human) ECs exposed to sepsis-associated mediators. ${ }^{46}$ Further, Yang et al reported correlation between the thickness of cancer cell glycocalyx and the uptake of gold nanocapsules, suggesting that a thinner glycocalyx allows more uptake. ${ }^{19}$ This suggests that the models utilized in this study, which have significantly reduced glycocalyx thickness, should lead to an impaired barrier function and a greater particle uptake. For both collapsed and degraded glycocalyx configurations, statistically significantly higher uptake of Alexa Fluor 647-conjugated PEG-AuNP was observed as expected. Compared to the control RFPEC monolayers with intact glycocalyx, RFPEC with collapsed glycocalyx took up 7.0 \pm 1 .6-fold more NPs, whereas RFPECs with degraded glycocalyx took up 6.1 \pm 0.4 fold more as indicated by the fluorescence intensity quantification (Figure 4A and B). Additionally, the two dysfunctional glycocalyx layers exhibited different AuNP distribution within the ECs: collapse due to the lack of serum which led to more AuNP being internalized within the cell body, while enzyme degradation showed AuNP primarily localizing within the glycocalyx (Figure 4C). Consistent results over five experiments suggest that the NP internalization that we observed is global and that the subcellular localization of the AuNP is statistically relevant. While others have reported a similar increase in nanoscale particle permeability for other cell types and conditions, ${ }^{18,34,47,48}$ to our knowledge, this is the first time the experiment has been conducted with nonactively targeted AuNP in RFPEC with the intracellular localization identified. Additionally, previously published studies did not attempt to repair the glycocalyx, but only compared the healthy glycocalyx to the degraded glycocalyx. ${ }^{18,23,34}$ Hence, exogenous HS was added back into the culture after HS degradation, in the hopes of observing a change in PEG-AuNP uptake from the degraded model.

\section{HS replacement restores glycocalyx blockage of PEG-AuNP entry in the EC}

Recovery of the impaired permeability from the degradation of the HS glycosaminoglycans was attempted by incubating the heparinase III-treated cells in media containing exogenous HS (DMEM with $59 \mu \mathrm{g} / \mathrm{mL} \mathrm{HS,} 10 \% \mathrm{FBS}$, and $1 \%$ penicillinstreptomycin) for 16 hours before fixation. The HS supplementation increased the overall glycocalyx thickness from $1.96 \pm 0.3$ to $2.41 \pm 0.3 \mu \mathrm{m}$, but it did not affect the overall glycocalyx coverage, which remained at $75.2 \% \pm 4.0 \%$ (Figure $2 \mathrm{D}$ and E). The HS component thickness was also partially restored, from $0.74 \pm 0.23$ to $1.21 \pm 0.31 \mu \mathrm{m}$, but not quite reaching the baseline thickness of $1.81 \pm 0.28 \mu \mathrm{m}$ (Figure $3 \mathrm{D}$ and $\mathrm{E}$ ). 
More importantly, the replacement increased HS coverage to $33.1 \% \pm 3.7 \%$ (Figure 3D and F). Compared to the healthy control of $75.2 \% \pm 10.6 \%$ and degraded coverage of $7.7 \% \pm 2.9 \%$, it is a significant improvement from $10 \%$ to $44 \%$ of the baseline measurements (Figure 3D and F). These results show a partial structural recovery of the glycocalyx layer with respect to HS after incubation of ECs with the exogenous HS-supplemented media.

There are several possible explanations for the observed partial but not complete HS and glycocalyx recovery after adding HS supplements. First, the concentration of HS used in the rat EC culture media was equal to HS concentrations measured in plasma from human clinical trials. ${ }^{49} \mathrm{HS}$ found in the human plasma should correlate to the amount of HS shed from the clinical trial participants' blood vessel walls and may not necessarily correlate to the HS degraded in our study. Additionally, HS concentration is different between species and concentration imbalance of sugars in the media can cause further glycocalyx damage. ${ }^{37,50}$ Quantification of HS produced by RFPEC and the amount shed through heparinase III treatment will improve dosage calculations for future experiments. Second, the exogenous HS was derived from porcine, which may cause a mismatch of physical characteristics with the RFPEC culture. The glycosaminoglycans differ from species to species not only in concentrations but also in patterns of sulfate groups on the sugar chains. ${ }^{5,6}$ The porcine HS may not be fully compatible with the RFPEC and alludes to the partial recovery, ${ }^{51}$ and in future studies the use of rat-derived HS may improve glycocalyx regrowth. Third, there is little control over how the exogenous HS is incorporated into the glycocalyx after addition to the culture. It can bind to proteoglycan sites that are exposed after enzyme cleavage, be entrapped within the glycocalyx mesh in a manner similar to albumin, or be endocytosed to increase the intracellular concentration of HS components rather than the cell surface concentration of HS. In the endogenous HS production pathway, the proteoglycans are synthesized in the ribosome and glycosylated in the Golgi apparatus, which are then exported to the cell membrane via Golgiand rough endoplasmic reticulum-regulated secretory vesicles. ${ }^{52}$ In future studies, it may be more advantageous to upregulate this endogenous HS production pathway via delivery of plasmids, rather than an exogenous route, to ensure the production of new HS at the cell membrane for a full glycocalyx recovery.

Despite the observed partial HS and glycocalyx recovery after adding exogenous HS, the barrier function was completely recovered with respect to $10 \mathrm{~nm}$ NPs. PEG-AuNP uptake studies in the glycocalyx-regenerated EC samples exhibited a staggering decrease in permeability compared to the two dysfunctional glycocalyx models (Figure 4A). The uptake of the NPs by these cells was measured to be a mere $1.3 \pm 0.3$-fold greater than the uptake by cells with healthy, intact glycocalyx (Figure 4B), and the confocal images showed almost no PEG-AuNP entrapment in the monolayer (Figure 4A). This fold change was significantly low compared to the Hep III-treated samples, demonstrating restored impedance of NP uptake into ECs in the presence of exogenous HS. Similar to the control, the localization plot showed a nonspecific distribution within the RFPEC culture (Figure 4C). While there are reports of increased NP uptake or interaction for dysfunctional or compromised glycocalyx on various cell types, to our knowledge none have recovered and reexamined the glycocalyx permeability. Although the glycocalyx was not thoroughly regenerated, this recovery of the barrier function points to a new approach in passive NP delivery to ECs within the circulatory system. Design of NP therapeutics such that they take advantage of this permeability changes under dysfunction in conjunction with active targeting can lead to higher targeting efficiency to the areas of interest and bypass the healthy vasculature.

\section{Conclusion}

Glycocalyx collapse and discontinuity have both been suggested to colocalize with vascular sites that are prone to cardiovascular disease. ${ }^{53}$ This study focused on taking glycocalyx conditions into consideration for scenarios of targeted, systemic NP-based drug delivery. Glycocalyx structure was modulated by serum deprivation or enzymatic cleavage of HS and subsequently supplementing exogenous HS, and probed using PEG-AuNP transport. The data show that the glycocalyx structure greatly affects the ability of PEG-AuNP to permeate ECs. In a healthy EC culture, the uptake of $10 \mathrm{~nm}$ particles was impeded by the endothelial glycocalyx. The dysfunction of the glycocalyx, both collapsed and degraded, led to an increase in NP uptake of over sixfold (Figure 4). This indicates a compromised barrier function of the glycocalyx and an additional mechanism for targeting NPs to vascular locations at risk for cardiovascular disease. Furthermore, the recovery of glycocalyx and the associated restoration of the protective function shown by the decrease in PEG-AuNP fluorescence indicate possibilities for nanomedicine-based glycocalyx therapeutics. In addition to (removed ) the size constraint of the nanocarrier design, regeneration of the glycocalyx can lead to particle rejection 
once healed, allowing the particles to target other locations downstream. While the AuNP do not support encapsulation and delivery of glycosaminoglycans, other types of particles can be formulated to specifically repair diseased glycocalyx. For example, physically delivering missing components or upregulating glycocalyx production via gene delivery are potential considerations. Glycocalyx repair would then prevent further particles from attaching and the remaining circulating particles can move downstream and to another area of dysfunction - this allows for a better distribution among the various diseased EC areas in vasculature. The PEG-AuNP used also have the potential to be improved by conjugating the functional PEGs with antibodies against endothelialspecific targets (we are referring to nonglycocalyx targets in this case), therapeutics that need to be delivered via the vasculature in an endothelial-specific manner, and imaging probes to enable in situ visualization of the NPs. The ability to deliver these NPs to dysfunctional and disease-prone ECs by exploiting glycocalyx behavior will significantly advance cardiovascular and other areas of drug delivery.

\section{Acknowledgments}

For the technical and financial support that was provided, the authors would like to thank the Northeastern University Office of the Provost; Northeastern University's Departments of Chemical Engineering, Physics, and Pharmaceutical Science; Northeastern University's Electronic Materials Research Institute; Dr Mia Thi of Albert Einstein College of Medicine; Dr Homa Homayoni; The National Institutes of Health (NIH) for the Mentored Career Development Award (K01-HL125499, granted to E Ebong); and the National Science Foundation (NSF) for the Nanomedicine Science and Technology Integrative Graduate Education and Research Traineeship (IGERT) program (DGE-096843, granted to Northeastern University).

\section{Disclosure}

The authors report no conflicts of interest in this work.

\section{References}

1. Fels J, Jeggle P, Liashkovich I, Peters W, Oberleithner H. Nanomechanics of vascular endothelium. Cell Tissue Res. 2014;355(3):727-737.

2. Pries AR, Secomb TW, Gaehtgens P. The endothelial surface layer. Pflugers Arch. 2000;440(5):653-666.

3. Weinbaum S, Zhang X, Han Y, Vink H, Cowin SC. Mechanotransduction and flow across the endothelial glycocalyx. Proc Natl Acad Sci U S A. 2003; 100(13):7988-7995.

4. Gamse G, Fromme HG, Kresse H. Metabolism of sulfated glycosaminoglycans in cultured endothelial cells and smooth muscle cells from bovine aorta. Biochim Biophys Acta. 1978;544(3):514-528.
5. Ihrcke NS, Wrenshall LE, Lindman BJ, Platt JL. Role of heparan sulfate in immune system-blood vessel interactions. Immunol Today. 1993; 14(10):500-505.

6. Oohira A, Wight TN, Bornstein P. Sulfated proteoglycans synthesized by vascular endothelial cells in culture. J Biol Chem. 1983;258(3): 2014-2021.

7. Tarbell JM, Ebong EE. The endothelial glycocalyx: a mechano-sensor and -transducer. Sci Signal. 2008;1(40):1-8.

8. Adamson RH, Clough G. Plasma proteins modify the endothelial cell glycocalyx of frog mesenteric microvessels. J Physiol. 1992;445: 473-486.

9. Loeven MA, Rops AL, Berden JH, Daha MR, Rabelink TJ, van der Vlag J. The role of heparan sulfate as determining pathogenic factor in complement factor H-associated diseases. Mol Immunol. 2015;63(2): 203-208.

10. Reitsma S, Slaaf DW, Vink H, van Zandvoort M, oude Egbrink MGA. The endothelial glycocalyx: composition, functions, and visualization. Pflugers Arch. 2007;454(3):345-359.

11. Alphonsus CS, Rodseth RN. The endothelial glycocalyx: a review of the vascular barrier. Anaesthesia. 2014;69(7):777-784.

12. Oberleithner $H$. Vascular endothelium: a vulnerable transit zone for merciless sodium. Nephrol Dial Transplant. 2014;29(2):240-246.

13. Tabas I, Garcia-Cardena G, Owens GK. Recent insights into the cellular biology of atherosclerosis. J Cell Biol. 2015;209(1):13-22.

14. Broekhuizen LN, Mooij HL, Kastelein JJ, Stroes ES, Vink H, Nieuwdorp M. Endothelial glycocalyx as potential diagnostic and therapeutic target in cardiovascular disease. Curr Opin Lipidol. 2009;20(1):57-62.

15. Nieuwdorp M, Meuwese MC, Vink H, Hoekstra JB, Kastelein JJ, Stroes ES. The endothelial glycocalyx: a potential barrier between health and vascular disease. Curr Opin Lipidol. 2005;16(5):507-511.

16. van den Berg BM, Nieuwdorp M, Stroes ES, Vink H. Glycocalyx and endothelial (dys) function: from mice to men. Pharmacol Rep. 2006; 58(Suppl):75-80.

17. Van Teeffelen JW, Brands J, Stroes ES, Vink H. Endothelial glycocalyx: sweet shield of blood vessels. Trends Cardiovasc Med. 2007;17(3): 101-105.

18. Atukorale PU, Yang YS, Bekdemir A, et al. Influence of the glycocalyx and plasma membrane composition on amphiphilic gold nanoparticle association with erythrocytes. Nanoscale. 2015;7(26):11420-11432.

19. Yang YS, Carney RP, Stellacci F, Irvine DJ. Enhancing radiotherapy by lipid nanocapsule-mediated delivery of amphiphilic gold nanoparticles to intracellular membranes. ACS Nano. 2014;8(9):8992-9002.

20. Smith SC Jr, Benjamin EJ, Bonow RO, et al. AHA/ACCF Secondary Prevention and Risk Reduction Therapy for Patients with Coronary and other Atherosclerotic Vascular Disease: 2011 update: a guideline from the American Heart Association and American College of Cardiology Foundation. Circulation. 2011;124(22):2458-2473.

21. Zhang L, Gu FX, Chan JM, Wang AZ, Langer RS, Farokhzad OC. Nanoparticles in medicine: therapeutic applications and developments. Clin Pharmacol Ther. 2008;83(5):761-769.

22. Kim BH, Hackett MJ, Park J, Hyeon T. Synthesis, characterization, and application of ultrasmall nanoparticles. Chem Materials. 2014;26(1): 59-71.

23. Brigger I, Dubernet C, Couvreur P. Nanoparticles in cancer therapy and diagnosis. Adv Drug Deliv Rev. 2002;54(5):631-651.

24. Adak AK, Li BY, Lin CC. Advances in multifunctional glycosylated nanomaterials: preparation and applications in glycoscience. Carbohydr Res. 2015;405:2-12.

25. Garrett IR, Gutierrez GE, Rossini G, et al. Locally delivered lovastatin nanoparticles enhance fracture healing in rats. JOrthop Res. 2007;25(10): 1351-1357.

26. Li H, Yu SS, Miteva M, et al. Matrix metalloproteinase responsive, proximity-activated polymeric nanoparticles for siRNA delivery. $A d v$ Funct Mater. 2013;23(24):3040-3052.

27. Torchilin VP. Multifunctional nanocarriers. Adv Drug Deliv Rev. 2012; 64(Supplement):302-315 
28. Freeman CG, Parish CR, Knox KJ, et al. The accumulation of circulating histones on heparan sulphate in the capillary glycocalyx of the lungs. Biomaterials. 2013;34(22):5670-5676.

29. Lu B, Xiong S-B, Yang H, Yin X-D, Chao R-B. Solid lipid nanoparticles of mitoxantrone for local injection against breast cancer and its lymph node metastases. Eur J Pharm Sci. 2006;28(1-2):86-95.

30. Chono S, Li S-D, Conwell CC, Huang L. An efficient and low immunostimulatory nanoparticle formulation for systemic siRNA delivery to the tumor. J Control Release. 2008;131(1):64-69.

31. Oishi M, Hayashi H, Iijima M, Nagasaki Y. Endosomal release and intracellular delivery of anticancer drugs using $\mathrm{pH}$-sensitive PEGylated nanogels. J Mater Chem. 2007;17(35):3720-3725.

32. Nishimura Y, Takeda K, Ezawa R, Ishii J, Ogino C, Kondo A. A display of $\mathrm{pH}$-sensitive fusogenic GALA peptide facilitates endosomal escape from a bio-nanocapsule via an endocytic uptake pathway. J Nanobiotechnol. 2014;12:11.

33. Paszek MJ, DuFort CC, Rossier O, et al. The cancer glycocalyx mechanically primes integrin-mediated growth and survival. Nature. 2014;511(7509):319-325.

34. Yang C, Liu Y, He Y, et al. The use of HA oligosaccharide-loaded nanoparticles to breach the endogenous hyaluronan glycocalyx for breast cancer therapy. Biomaterials. 2013;34(28):6829-6838.

35. Lange T, SamatovTR, Tonevitsky AG, SchumacherU.Importance ofaltered glycoprotein-bound N- and O-glycans for epithelial-to-mesenchymal transition and adhesion of cancer cells. Carbohydr Res. 2014;389: $39-45$.

36. Kumar R, Korideck H, Ngwa W, et al. Third generation gold nanoplatform optimized for radiation therapy. Transl Cancer Res. 2013;2(4): 228-239.

37. Ebong EE, Macaluso FP, Spray DC, Tarbell JM. Imaging the endothelial glycocalyx in vitro by rapid freezing/freeze substitution transmission electron microscopy. Arterioscler Thromb Vasc Biol. 2011;31(8): 1908-1915.

38. Zeng Y, Ebong EE, Fu BM, Tarbell JM. The structural stability of the endothelial glycocalyx after enzymatic removal of glycosaminoglycans. PLoS One. 2012;7(8):e43168.

39. Hainfeld JF, Dilmanian FA, Slatkin DN, Smilowitz HM. Radiotherapy enhancement with gold nanoparticles. J Pharm Pharmacol. 2008;60(8): 977-985.

40. Popovtzer R, Agrawal A, Kotov NA, et al. Targeted gold nanoparticles enable molecular CT imaging of cancer. Nano Lett. 2008;8(12): 4593-4596.
41. Shukla R, Bansal V, Chaudhary M, Basu A, Bhonde RR, Sastry M. Biocompatibility of gold nanoparticles and their endocytotic fate inside the cellular compartment: a microscopic overview. Langmuir. 2005; 21(23):10644-10654.

42. Zeng Y, Tarbell JM. The adaptive remodeling of endothelial glycocalyx in response to fluid shear stress. PLoS One. 2014;9(1):e86249.

43. Schneeberger EE, Hamelin M. Interaction of serum proteins with lung endothelial glycocalyx: its effect on endothelial permeability. Am J Physiol Heart Circul Physiol. 1984;247(2):H206-H217.

44. Henry CB, Duling BR. Permeation of the luminal capillary glycocalyx is determined by hyaluronan. Am J Physiol. 1999;277(2 Pt 2): H508-H514.

45. van den Burg MM, Eizema K, de Graaf-Roelfsema E, et al. Effects of acute exercise and long-term exercise on total $\mathrm{Na}+\mathrm{K}+$-ATPase content and $\mathrm{Na}+, \mathrm{K}+-\mathrm{ATPase}$ isoform expression profile in equine muscle. Am J Vet Res. 2009;70(7):895-901.

46. Wiesinger A, Peters W, Chappell D, et al. Nanomechanics of the endothelial glycocalyx in experimental sepsis. PLoS One. 2013;8(11): e80905.

47. Liu J, Agrawal NJ, Calderon A, Ayyaswamy PS, Eckmann DM, Radhakrishnan R. Multivalent binding of nanocarrier to endothelial cells under shear flow. Biophys J. 2011;101(2):319-326.

48. Zhu J, Xue J, Guo Z, Zhang L, Marchant RE. Biomimetic glycoliposomes as nanocarriers for targeting P-selectin on activated platelets Bioconjug Chem. 2007;18(5):1366-1369.

49. Rehm M, Bruegger D, Christ F, et al. Shedding of the endothelial glycocalyx in patients undergoing major vascular surgery with global and regional ischemia. Circulation. 2007;116(17):1896-1906.

50. Piest M, Ankone M, Engbersen JF. Carbohydrate-interactive pDNA and siRNA gene vectors based on boronic acid functionalized poly(amido amine)s. J Control Release. 2013;169(3):266-275.

51. Ernst S, Langer R, Cooney CL, Sasisekharan R. Enzymatic degradation of glycosaminoglycans. Crit Rev Biochem Mol Biol. 1995;30(5): $387-444$.

52. Varki A, Cummings RD, Esko JD. Essentials of Glycobiology. Cold Spring Harbor, NY: Cold Spring Harbor Laboratory Press; 2009.

53. Lewis JC, Taylor RG, Jones ND, St Clair RW, Cornhill JF. Endothelial surface characteristics in pigeon coronary artery atherosclerosis. I. Cellular alterations during the initial stages of dietary cholesterol challenge. Lab Invest. 1982;46(2):123-138.
International Journal of Nanomedicine

\section{Publish your work in this journal}

The International Journal of Nanomedicine is an international, peerreviewed journal focusing on the application of nanotechnology in diagnostics, therapeutics, and drug delivery systems throughout the biomedical field. This journal is indexed on PubMed Central,

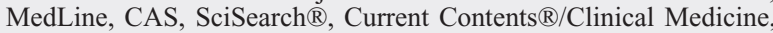

\section{Dovepress}

Journal Citation Reports/Science Edition, EMBase, Scopus and the Elsevier Bibliographic databases. The manuscript management system is completely online and includes a very quick and fair peer-review system, which is all easy to use. Visit http://www.dovepress.com/ testimonials.php to read real quotes from published authors. 